\title{
The Effects of Squats and Jump Squats on Mechanical Work and Energy Expenditure
}

Shawn N. Munford, Michael L. Rossetti, Gavin L. Moir, Brandon W. Snyder, Shala E. Davis, FACSM. East Stroudsburg University of Pennsylvania, East Stroudsburg, PA.

PURPOSE: To investigate the effects of two non-ballistic squat and two ballistic jump squat protocols performed over multiple sets on the total mechanical work performed and oxygen uptake. METHODS: In a counterbalanced cross-over design, 11 resistance-trained men (age: $21.9 \pm 1.8$ years; height: $1.79 \pm 0.05 \mathrm{~m}$; mass: $87.0 \pm 7.4 \mathrm{~kg}$ ) attended four testing sessions during a three week period where they performed multiple sets of squats and jump squats with a load equivalent to $30 \%$ 1-repeititon maximum under one of the following conditions: 1) three sets of four non-ballistic repetitions $(30 \mathrm{~N}-\mathrm{B}), 2)$ three sets of four non-ballistic repetitions with a 3 -second pause between the eccentric and concentric phases (30PN-B), 3) three sets of four ballistic repetitions $(30 \mathrm{~B}), 4)$ three sets of four ballistic repetitions with a 3 -second pause between the eccentric and concentric phases (30PB). Force plates and a 3-D motion analysis system were used to determine the total mechanical work performed during each session while a portable gas analysis system was used to collect expired gases. RESULTS: Total mechanical work performed during each set was significantly greater during $30 \mathrm{~B}$ compared to $30 \mathrm{~N}-\mathrm{B}$ (mean difference [MD]: 7,792 J, p<0.001, effect size [ES]: 1.88) and 30PN-B (MD: 7,749 J, p<0.001, ES: 1.89), while that during 30PB was significantly greater than that during both $30 \mathrm{~N}-\mathrm{B}$ (MD: 7,488 J, p<0.001, ES: 1.89) and 30PN-B (MD: 7,445 J, p<0.001, ES: 1.90). Energy expenditure during each set was significantly greater during the $30 \mathrm{~B}$ condition compared to the $30 \mathrm{~N}-\mathrm{B}$ (MD: $13,983 \mathrm{~J}, \mathrm{p}<0.001$, ES: 1.08) and the 30PN-B (MD: 11,326 J, p=0.001, ES: 0.92). Energy expenditure during $30 \mathrm{~PB}$ was also significantly greater than that during $30 \mathrm{~N}-\mathrm{B}$ (MD: $12,615 \mathrm{~J}$, $\mathrm{p}=0.001$, ES: 1.04) and 30PN-B (MD: 9,958 J, p=0.006, ES: 0.86). Furthermore, energy expenditure during set 1 was significantly greater than that during set 2 (MD: 6,840 J, p $<0.001$, ES: 0.64) and set 3 (MD: 8,070 J, p<0.001, ES: 0.75). CONCLUSION: Ballistic resistance training exercises may represent a more effective metabolic stimulus compared to traditional resistance training exercises and a pause inserted between the eccentric and concentric phases has little effect. 